\title{
Electromagnetic Band Gap Coupled Microstrip Antenna for UWB Applications
}

\author{
Aijaz Ahmed ${ }^{1}$, Yogesh ${ }^{2}$ \\ ${ }^{1,2}$ (Dept. of ECE, ASET/ Amity University, India)
}

\begin{abstract}
In this paper Electromagnetic Band Gap structure with microstrip antenna has been proposed. Many small and compact periodic EBGs have been investigated to design the antenna. Simulation is done on the HFSS software tool. The antenna is designed on the $21 \times 31 \mathrm{~mm}$ FR4 substrate with the dielectric constant of 4.4 . The designed antenna is designed to resonate at $8 \mathrm{GHz}$ frequency. The designed antenna is having the peak return loss of $49.87 \mathrm{~dB}$ at $7.7 \mathrm{GHz}$ frequency with the bandwidth of $4.7 \mathrm{GHz}$ from $5.3 \mathrm{GHz}$ to $10 \mathrm{GHz}$, thus has the wide range of applications in ultra wideband communication.
\end{abstract}

Keywords-EBG, microstrip antenna, patch antenna, UWB, wideband antenna.

\section{INTRODUCTION}

The features of electromagnetic band gap structure are suppressing unwanted substrate modes and acting as an artificial magnetic ground plane. The EBG structures are also used to improve the antenna performance as radiation patterns and to minimize the side and back lobe levels [1,2]. However, most researchers only study the EBG effects on a single microstrip antenna element, and to the best of our knowledge there are no comprehensive results reported for antenna arrays. Microstrip antennas have been used widely and continue to be popular in commercial applications due to their light weight, low profile, low cost and ease of integration with MMIC fabrication technology. The major limitations of a patch antenna on a high dielectric constant substrate are its low efficiency due to surface wave loss and inherently narrow bandwidth. Frequencyselective surface have currently attached considerable attention due to the growing interest in utilizing electromagnetic band-gap (EBG) structure. The particular role of an electromagnetic band gap (EBG) technique is that it can be applied to improve performance of a planar antenna designed on a high dielectric material. By loading the EBGs periodically on the substrate, a band gap can be created for frequencies around the operating frequency of the antenna. Such structure can stop the propagation of surface waves, which can be excited along the high dielectric substrate material supporting MMIC components. By doing this more power can be coupled to a space wave instead of being wasted in the substrate. In general, EBGs can be realized as 1-D (one dimensional), 2-D or 3-D. In the proposed paper 2-D EBG structure is carried out.

\section{ChARACTERISTICS OF EBG STRUCTURE}

This structure has frequency range where the surface impedance is very high. The equivalent LC circuit acts as a two-dimensional electric filter in this range of frequency to block the flow of the surface waves. The central frequency of the band gap is shown in equation 1. The inductor L results from the current flowing through the via, and the capacitor $\mathrm{C}$ due to the gap effect between the adjacent patches. Thus, the approach to increase the inductance or capacitance will naturally result in the decrease of band-gap position $[3,4,5,6 \& 7]$.

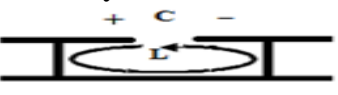

Fig. 1: 2D EBG Structure

Central frequency of Band Gap is given by:

$f_{c}=1 /(2 \pi \sqrt{L C})$

Where:

$$
\begin{aligned}
& \mathrm{L}=\mu_{0} \mathrm{~h} \\
& C=W \varepsilon_{o} \frac{\left(\varepsilon_{r}+1\right)}{\pi} \cosh ^{-1}\left(\frac{2 W+g}{g}\right)
\end{aligned}
$$

\section{ANTENNA STRUCTURE}

In this paper a rectangular patch antenna has been designed with the EBG Structure. The antenna is designed on FR4 with dielectric constant 4.4 and with the thickness of $1.67 \mathrm{~mm}$ with the width and the length of $21 \mathrm{~mm}$ and 
$31 \mathrm{~mm}$ respectively. The ground plane material is taken as copper. The structure of antenna is shown in fig.2(a). fig.2 (b) shows the EBG surface specification. The gap of the EBG patches is $1 \mathrm{~mm}$ in both sides and each patch block is having the cross section of $4 \mathrm{x} 4 \mathrm{~mm}$. Each $4 \mathrm{x} 4$ patch is connected to copper ground plane with the help of via through the substrate. The diameter of each via is $1 \mathrm{~mm}$.

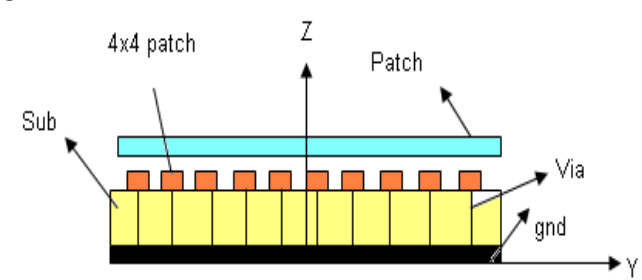

Fig. 2(a): Antenna structure

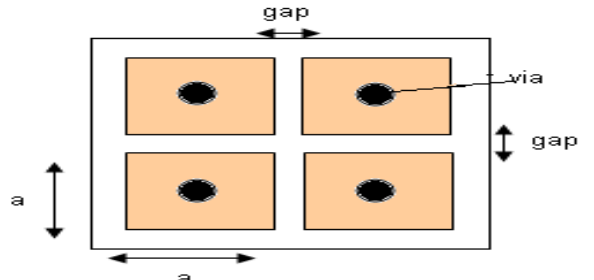

(b) EBG specifications

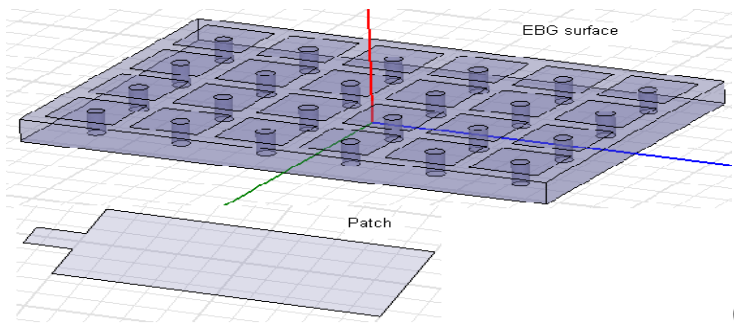

( c)

IV. RESULTS AND DISCUSSIONS

In figure 3 Return loss with and with EBG structure is shown. The antenna is designed for the resonance frequency of $8 \mathrm{GHz}$. The figure shows that the antenna is having the peak return loss of $49.87 \mathrm{~dB}$ at 7.7GHz. Antenna is having a wide band range of $5.3 \mathrm{GHz}$ to $10 \mathrm{GHz}$ (Bandwidth $4.7 \mathrm{GHz}$ ). fig. 4 shows the VSWR plot of the antenna, from the figure it is clear that the VSWR in the radiating band of the antenna is lying between 1 and 2 which is desirable. Figure 6 and 7 shows the radiation pattern of the antenna with respect to theta and phi respectively for two different placement angles of 0 degree and 90 degree. The axial ratio variation for different values of phi angle is shown in figure 8 .

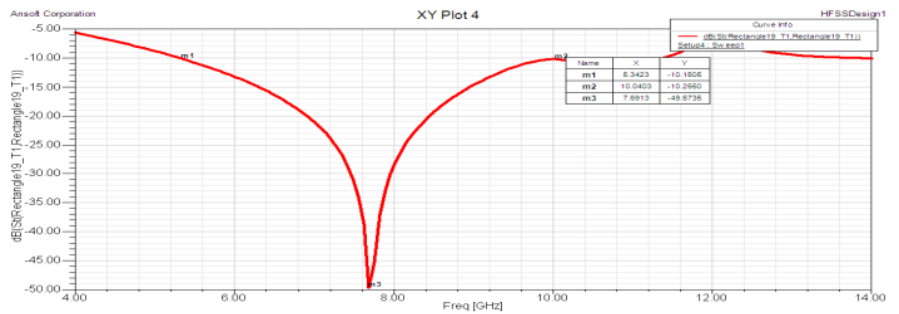

Fig.4 Return loss Characteristic of EBG antenna

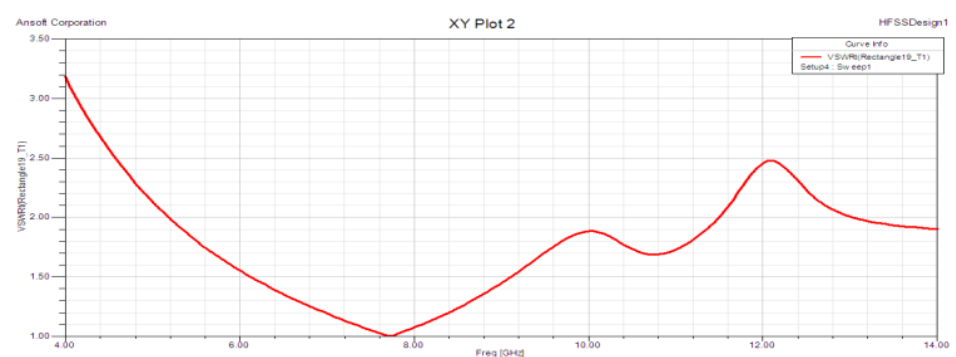

Fig.5 VSWR plot of the antenna

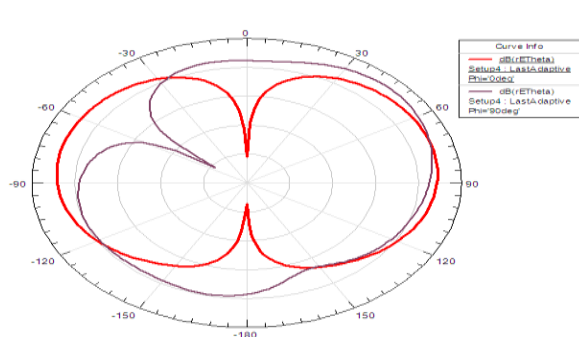

Fig.6 Theta pattern of antenna

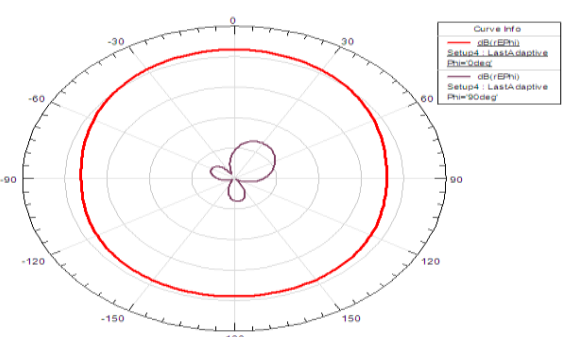

Fig.7 Phi pattern of antenna 


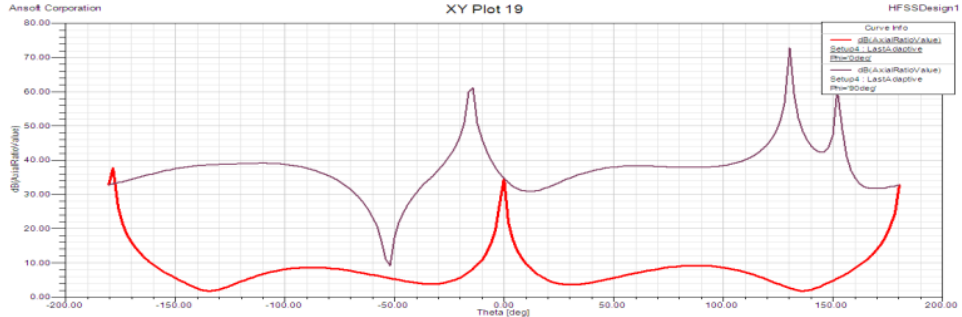

Fig.8 Axial ratio of antenna

V.

CONCLUSION

A simple rectangular antenna with EBG structure of $4 x 4 \mathrm{~mm}$ periodic patches has been proposed. The antenna is providing the return loss of approximately $50 \mathrm{~dB}$ which is very good for microstrip antennas. The designed antenna is having the wide band of $4.7 \mathrm{GHz}$ lying in the Ultra wide band range, thus having a wide range of applications in UWB communication.

\section{REFERENCES}

[1]. Y. Fu and N. Yuan, "Elimination of scan blindness in phased array of microstrip patches using electromagnetic bandgap materials," IEEE Antennas Wireless Propag. Lett., vol. 3, pp. 64-65, 2004.

[2]. J. S. Colburn and Y. Rahmat-Samii, "Patch antennas on externally perforated high dielectric constant substrates," IEEE Trans. Antennas Propag., vol. 47, no. 12, pp. 1785-1794, Dec. 1999.

[3]. Pirhadi, A., M. Hakkak, and F. Keshmiri, "Bandwidth enhancement of the probe fed microstrip antenna using frequency selective surface as electromagnetic bandgap superstrate," Progress In Electromagnetics Research, PIER 61, 215-230, 2006.

[4]. Pirhadi, A., F. Keshmiri, and M. Hakkak, "Design of dualband low profile high directive EBG resonator antenna, using single layer frequency selective surface superstrate," IEEE APS/USNC/URSI Int. Symp., Albuquerque, New Mexico, USA, July 9-14, 2006.

[5]. Lee, Y. J., J. Yeo, R. Mittra, and W. S. Park, "Application of electromagnetic bandgap (EBG) superstrates with controllable defects for a class of patch antennas as spatial angular filters," IEEE Trans. Antennas and Propag., Vol. AP-53, No. 1, 224-234, Jan. 2005.

[6]. G. Cakir and L. Sevgi, "Design of a novel microstrip electromagnetic band-gap (EBG) structure," Microw. Opt.Technol Lett., vol. 46, pp. 399-401, 2005.

[7]. F. Yang and Y. Rahmat-Samii, "Microstrip antennas integrated with electromagnetic band-gap (EBG) structures: A low mutual coupling design for array applications," IEEE Trans. Antennas Propag., vol. 51, no. 10, pp. 2936-2946, Oct. 2003.

[8]. Ansoft Corporation, HFSS 14. 